\title{
Structure and dynamical steady state of the peeling mode in edge-localized mode-free, high- confinement tokamak plasmas
}

Kensaku Kamiya ( $\square$ kamiya.kensaku@qst.go.jp )

National Institute for Quantum and Radiological Science and Technology

Kimitaka Itoh

Chubu University

Nobuyuki Aiba

National Institutes for Quantum and Radiological Science and Technology

Naoyuki Oyama

National Institute for Quantum and Radiological Science and Technology

Mitsuru Honda

National Institute for Quantum and Radiological Science and Technology

Akihiko Isayama

National Institute for Quantum and Radiological Science and Technology

\section{Article}

Keywords: edge-localized modes, low-n, edge-harmonic oscillations, tokamak plasmas

Posted Date: January 4th, 2021

DOl: https://doi.org/10.21203/rs.3.rs-121450/v1

License: (9) (i) This work is licensed under a Creative Commons Attribution 4.0 International License. Read Full License

Version of Record: A version of this preprint was published at Communications Physics on June 17th, 2021. See the published version at https://doi.org/10.1038/s42005-021-00644-x. 


\title{
Structure and dynamical steady state of the peeling mode in edge-localized mode-free, high-confinement tokamak plasmas
}

\author{
K. Kamiya ${ }^{1 *}$, K. Itoh ${ }^{2}$, N. Aiba ${ }^{1}$, N. Oyama ${ }^{1}$, M. Honda ${ }^{1}$, and A. Isayama ${ }^{1}$

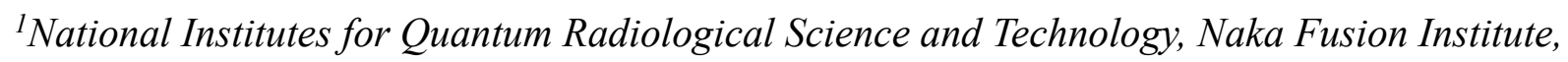 \\ Naka, Ibaraki, Japan \\ ${ }^{2}$ Chubu University, Kasugai, Aichi, Japan \\ *email: kamiya.kensaku@qst.go.jp
}

Explosive dynamical events in controlled-nuclear-fusion devices (known as edge-localized modes) display many similarities to solar-flare events on the sun, revealing a new connection between laboratory plasma physics and astronomy. However, to date there has been no direct evidence for the peeling mode structure, due to the lack of decisive diagnostics. Here we report the first evidence for the structure and dynamical steady state of a peeling mode for low- $n$ edge-harmonic oscillations (EHOs) in the quiescent $\mathrm{H}$-mode. EHOs are dominated by the fundamental mode $\left(1 \mathrm{f}_{\mathrm{EHO}}\right)$ at both the low- and high-field sides. $1 \mathrm{f}_{\mathrm{EHO}}$ edge perturbations are confirmed to have kink parity and exhibit the frozen-in-condition predicted by a linear stability analysis. The envelope signal of the $1 \mathrm{f}_{\mathrm{EHO}}$ mode exhibits repeated cycles of growth and damping to the order of a few hundred $\mathrm{Hz}$ associated with small changes in an edge gradient, and results are quantitatively consistent with a limit-cycleoscillation model. 
Studies of explosive dynamical events in controlled-nuclear-fusion devices (known as edge-localized modes $(E L M s)^{1-3}$ ) display many similarities to solar-flare events on the sun, revealing a new connection between laboratory plasma physics and astronomy ${ }^{4-5}$. Improved understanding of the common physics of ELMs and solar/stellar flares not only can contribute to our intellectual understanding of plasma physics, but also can improve the controllability of burning plasmas in selfregulating, combined/complex next-step devices such as ITER $^{6-7}$ and $\mathrm{DEMO}^{8-9}$. In particular, highconfinement-mode (H-mode) operation, which has a high pedestal pressure, may be impossible without a proper physics understanding of the "peeling" mode (and/or coupled peeling-ballooning modes). This seems essential for predicting the ELM stability limit, because the instability is driven by the pressure gradient and the parallel current (the so-called bootstrap current) ${ }^{10-13}$.

The quiescent $\mathrm{H}$-mode (QH-mode) regime was discovered first in DIII-D ${ }^{14}$, and it was subsequently reproduced in JT-60U ${ }^{15}$ and ASDEX-Upgrade/JET ${ }^{16}$, in which ELMs are replaced by continuous MHD oscillations (the so-called edge-harmonic oscillations, EHOs). Theoretical models, together with experimental support, predict that the QH-mode edge condition is always near the low- $n(\leq 5)$ boundary of the peeling mode instability ${ }^{17-20}$. However, to date there has been no direct evidence for the structure of the peeling mode, due to the lack of decisive diagnostics. This is especially true for fluctuation measurements at the high-field-side (HFS) of the tokamak edge region, and measurements have been limited solely to the low-field-side (LFS).

To identify the peeling mode, we present here the following five criteria for the associated EHOs: They must (1) be lower- $n$ modes $(\leq 1,2,3 \ldots)$, (2) have a mode structure that extends to all of the plasma surface (i.e., not just be localized at the LFS), (3) result in perturbation structures that are close to the edge current and/or corresponding eigenmode, (4) have "kink" parity (rather than "tearing" parity), and (5) be EHOs that are sustained for long times; i.e., times comparable to the currentdiffusion time in the edge region. New information about the dynamical steady state that occurs during the QH-mode is also given. The envelope signal of the dominant fundamental mode of the EHOs exhibits repeated cycles of growth and damping on the order of a few milliseconds that are associated with small changes in the pedestal gradient, and the results are quantitatively consistent with a limit-cycle-oscillation model.

\section{Results}

Observation of low-n EHOs at both the LFS and HFS. Figure 1 (a)-(d) shows that the dominant fundamental mode of the EHOs ( $1 \mathrm{f}_{\mathrm{EHO}}$ — —obtained from various diagnostics measured at different poloidal (toroidal) angles - occurs at a frequency $\sim 10 \mathrm{kHz}$ during a stationary QH-mode phase without large ELMs, keeping the plasma density constant. The magnetic diagnostics identify this $1 \mathrm{f}_{\mathrm{EHO}}$ mode as $n=2$. This QH-mode phase-with continuous EHOs $\left(\tau_{\mathrm{EHOs}} \geq 3 \mathrm{~s}\right)$-is sustained with 
a neutral-beam-injection power $\mathrm{P}_{\mathrm{NBI}}=14 \mathrm{MW}$ (4 MW counter-tangential, $5 \mathrm{MW}$ co-perpendicular, and $5 \mathrm{MW}$ counter-perpendicular injections) for $\mathrm{t}=4.5-7.5 \mathrm{~s}$ (Fig. 1d). This is significantly longer than the current-diffusion time in the edge region $\left[\tau_{\mathrm{R}} \sim \boldsymbol{O}(1 \mathrm{~s})\right.$ for $\left.\mathrm{T}_{\mathrm{e}, \mathrm{sep}} \sim 200 \mathrm{eV}\right]$.
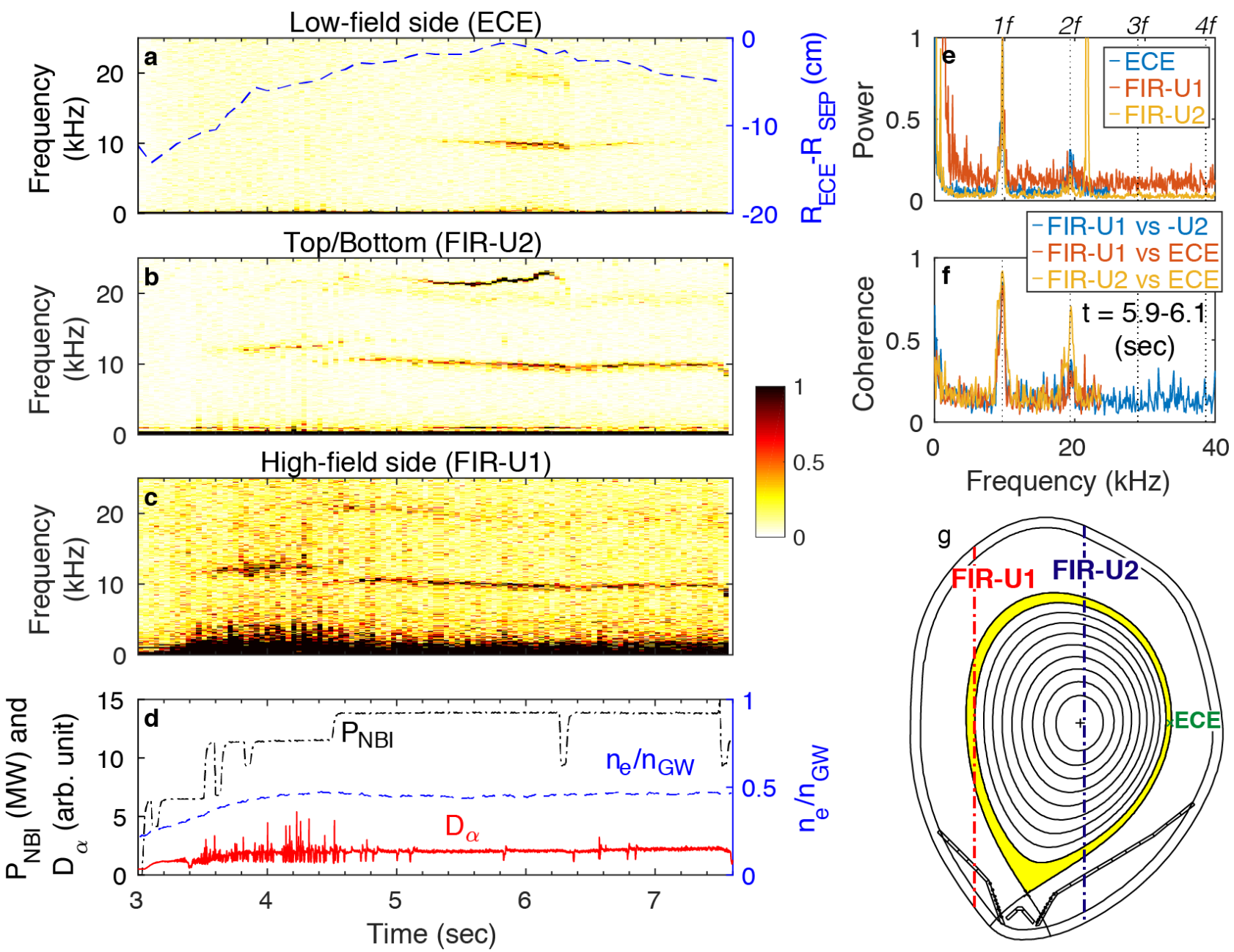

Figure 1 Quiescent H-mode shot (discharge E042870). Fourier analysis of signals (normalized at $\mathrm{t}=6.0 \mathrm{~s}$ ) (a) from the ECE at the LFS, together with its measurement position relative to the separatrix, and from the FIR laser interferometers for (b) the Top/Bottom (FIR-U2) and (c) the HFS (FIR-U1) chords. (d) The total NBI power, $D_{\alpha}$ emission from the divertor, and line-averaged electron density normalized by the Greenwald density. The frequency dependences of (e) the normalized power spectra for the ECE, FIR-U1, and -U2 and (f) their respective coherences evaluated at $\mathrm{t}=5.9-6.1 \mathrm{~s}$. (g) Magneto-hydrodynamic equilibrium (from the FBEQU-code) and the diagnostic locations at $\mathrm{t}=6.0 \mathrm{~s}$.

As illustrated in Figs. 1 (a)-(c) and (e)-(f), the spectra for the ECE, FIR-U1, and -U2 show that they manifest high coherence among them at the frequency $1 \mathrm{f}_{\mathrm{EHO}} \sim 10 \mathrm{kHz}(n=2)$. This observation suggests that the $1 \mathrm{f}_{\mathrm{EHO}}$ mode has a uniform mode structure over the entire circumference of the peripheral region of the plasma (Fig. 1g), and hence it is more likely to be a peeling mode (i.e., it is non-ballooning at the LFS). On the other hand, the power (and coherence) for the $2^{\text {nd }}$-harmonic 
component that exists at the frequency $2 f_{E H O} \sim 20 \mathrm{kHz}(n \sim 4)$ becomes weaker than that of the $1 \mathrm{f}_{\mathrm{EHO}}$ mode, and there are no higher-order modes. It is worth noting that the power spectrum of the EHOs observed in the QH-mode phase at JT-60U is characterized by a dominant fundamental MHD mode rather than containing strong higher-order harmonics (as seen in other devices ${ }^{14}$ ), which suggests weak nonlinearity (i.e., it is a quasi-linear mode).

Effects of GAPOUT and GAPIN values on ELMy/QH operational regimes. The GAPOUT value needed to access the stationary QH-mode regime in E042870 was determined by sweeping the edge plasma slowly in another discharge (i.e., E042868), with the cross-sectional shape of the plasma remaining fixed; hence, the GAPIN value is inversely correlated with the GAPOUT value. Here, the GAPOUT/GAPIN values are defined by the distance between the last closed flux surface and the wall at the outboard/inboard mid-plane.

Figure 2 (a) and (b) (discharge E042868) demonstrates an extensive gap scan experiment under experimental conditions almost identical to those of discharge E042870 (as shown in Fig. 1), except for the GAPOUT/GAPIN values. We observed small and frequent ELMs (f $\mathrm{fLM}_{2} \sim 100-300 \mathrm{~Hz}$ ) before the gap scan $(\mathrm{t} \leq 6.0 \mathrm{~s}$ ), with a configuration having a wider GAPOUT value of $\sim 38 \mathrm{~cm}$ (and a narrower GAPIN value of $\sim 33 \mathrm{~cm}$ ). During the gap scan toward narrower values of GAPOUT (and wider values of GAPIN), the frequencies of the ELMs decrease to $\mathrm{fELM}_{\mathrm{EM}} \sim 10 \mathrm{~Hz}$ or less, while accessing the QH-mode regime. Note that the GAPOUT/GAPIN values at $\mathrm{t}=6.5 \mathrm{~s}$ for discharge E042868 almost match those of discharge E042870.
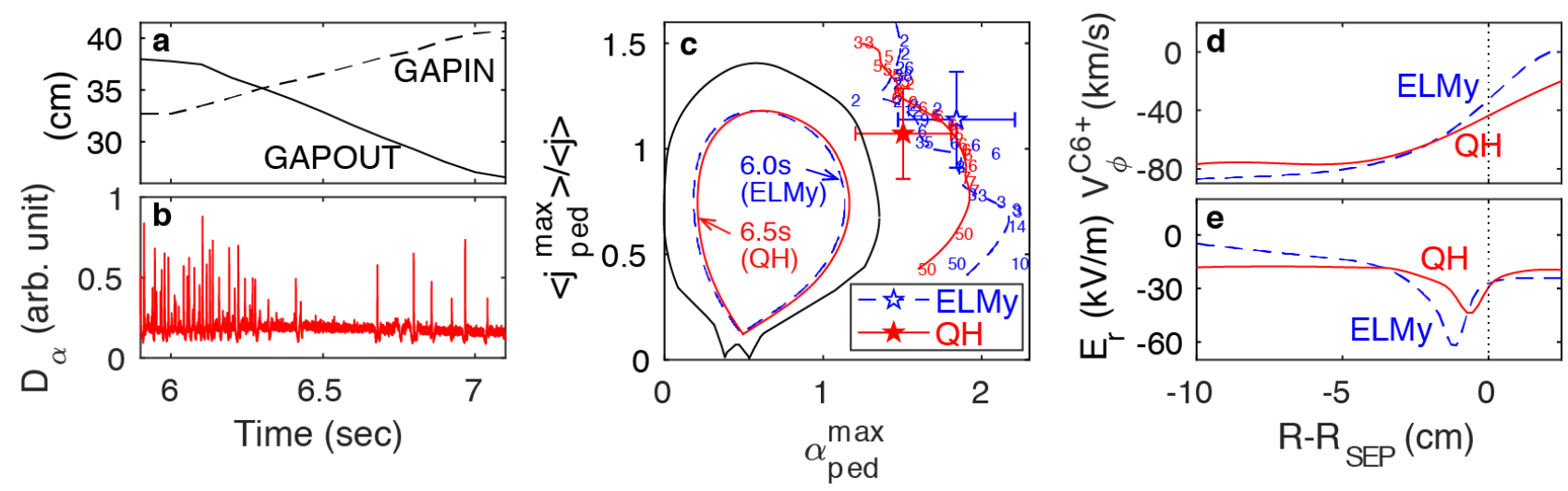

Figure 2 Transition from ELMy to the QH-mode in a gap scan during discharge E042868. (a) GAPOUT and GAPIN, and (b) $D_{\alpha}$ emission from the divertor. Comparisons between $\mathrm{t}=6.0 \mathrm{~s}$ (ELMy: blue dashed line) and $6.5 \mathrm{~s}$ (QH: red solid line) for (c) the MHD stability diagram in the normalized pedestal current $\left(j_{\text {ped. }}{ }^{\max } /<j>\right.$ ) and pressure $\left(\alpha_{\text {ped. }}{ }^{\text {max }}\right.$ ) plane, showing the MHD equilibria, and the radial profiles of $(\mathrm{d})$ the toroidal flow and (e) the radial electric field.

This observation suggests that the condition of a locally wide GAP only at the LFS seems to be 
insufficient for accessing the stationary QH-mode regime. In any case, this is a necessary condition (known to be one of the "control knobs" for accessing the QH-regime ${ }^{14}$ ). By comparison with other devices, the GAPOUT value seems to be wide enough for this ELMy phase in the pre-QH phase $(\mathrm{t} \leq$ $6.4 \mathrm{~s})$ to occur, while the ELMs reappear in the post-QH phase ( $\mathrm{t} \geq 6.7 \mathrm{~s})$ as the GAPOUT value becomes narrower (but, still wider than that of other devices) as is well known. This was pointed out in Ref. 15, which discussed the role of an optimized GAPOUT condition. On the other hand, the conditions of non-local GAPs at both the LFS and the HFS seem to be more important for the excitation of EHOs that have uniform mode structures over the entire circumference, absent the inhibition expected from a peeling mode structure. This observation is still challenging for the theoretical understanding of the conditions necessary and sufficient for accessing the QH-mode regime.

The theory of the stability of peeling-ballooning (kink) modes at the plasma edge allows us to confirm one of the peeling mode features for the EHOs in the QH-mode. As illustrated in Fig. 2 (c), the QH-mode data from JT-60U are consistent with the stability predictions of the theory, which have been discussed in previous publications from DIII-D ${ }^{21-23}$, because the operating points for the $\mathrm{QH}$ (and/or ELMy) phase are on or below (up to) the low- $n$ peeling boundary to within the experimental error bars. On the other hand, the dependence of the marginal-stability boundary on the pressure gradient $\left(\alpha_{\text {ped. }}\right)$ for JT-60U is stronger than that for DIII-D, suggesting that these two devices have different mechanisms for accessing the QH-mode regime. Furthermore, the radial structures of the toroidal flow $\left(V_{\phi}^{C 6+}\right)$ and of the radial electric field $\left(E_{r}\right)$ in the QH-mode phase at the pedestal region are observed to become slightly weaker than those in the ELMy phase (Figs. 2d and e), suggesting that a strong $\mathrm{E} \times \mathrm{B}$ shear flow is unnecessary. Instead, the appearance of EHOs seems to be strongly influenced by the optimization of the GAPs, as described above. Indeed, the phase transition from ELMy to the QH-mode occurs solely due to changes in the GAPs, while other external parameters are kept fixed (e.g., the cross-sectional shape of the plasma, its density, and the external input torque).

Radial structure of the peeling mode. Figure 3 demonstrates another important feature of the peeling mode structure for the EHOs in the QH-mode. Fluctuations in the normalized electrontemperature of the $1 \mathrm{f}_{\mathrm{EHO}}$ mode, as measured by the ECE with a bandpass filter $\left(9 \leq \mathrm{f}_{\mathrm{BPF}} \leq 10 \mathrm{kHz}\right)$ are observed to reach a local maximum value at $\mathrm{R}-\mathrm{R}_{\mathrm{SEP}} \sim-1 \mathrm{~cm}$, at which the pressure and current profiles have their own steep gradients at the pedestal (Figs. 3a-c). The envelope of the poloidal Fourier components $(n=2)$ of the linear eigenfunction, $\mathrm{R}_{\mathrm{e}}\left(\xi_{\mathrm{r}}\right)$, obtained with the ideal MHD code MARG2D ${ }^{24}$, exhibits multiple peak values, which decrease in amplitude from the separatrix to the core region. It is important that the product of the linear eigenfunction times the inverse gradient scale length $-R_{e}\left(\xi_{r}\right) \times L_{T_{e}}^{-1}\left(\equiv-\frac{\nabla T_{e}}{T_{e}}\right)$ - seems to be reasonably consistent with the radial structure of the 
$1 \mathrm{f}_{\mathrm{EHO}}$ mode, exhibiting the so-called "frozen-in" condition of the MHD mode (i.e., the field lines are frozen into the plasma and have to move along with it) ${ }^{25-26}$. This is one of the criteria for identifying the peeling mode. Furthermore, the radial structure of the relative phase difference between the ECE and the magnetic diagnostics (i.e., the saddle coil located on the vacuum vessel) is observed to be almost constant radially, exhibiting a non-tearing type of kink parity of the mode, as illustrated in Fig. $3(d)$.

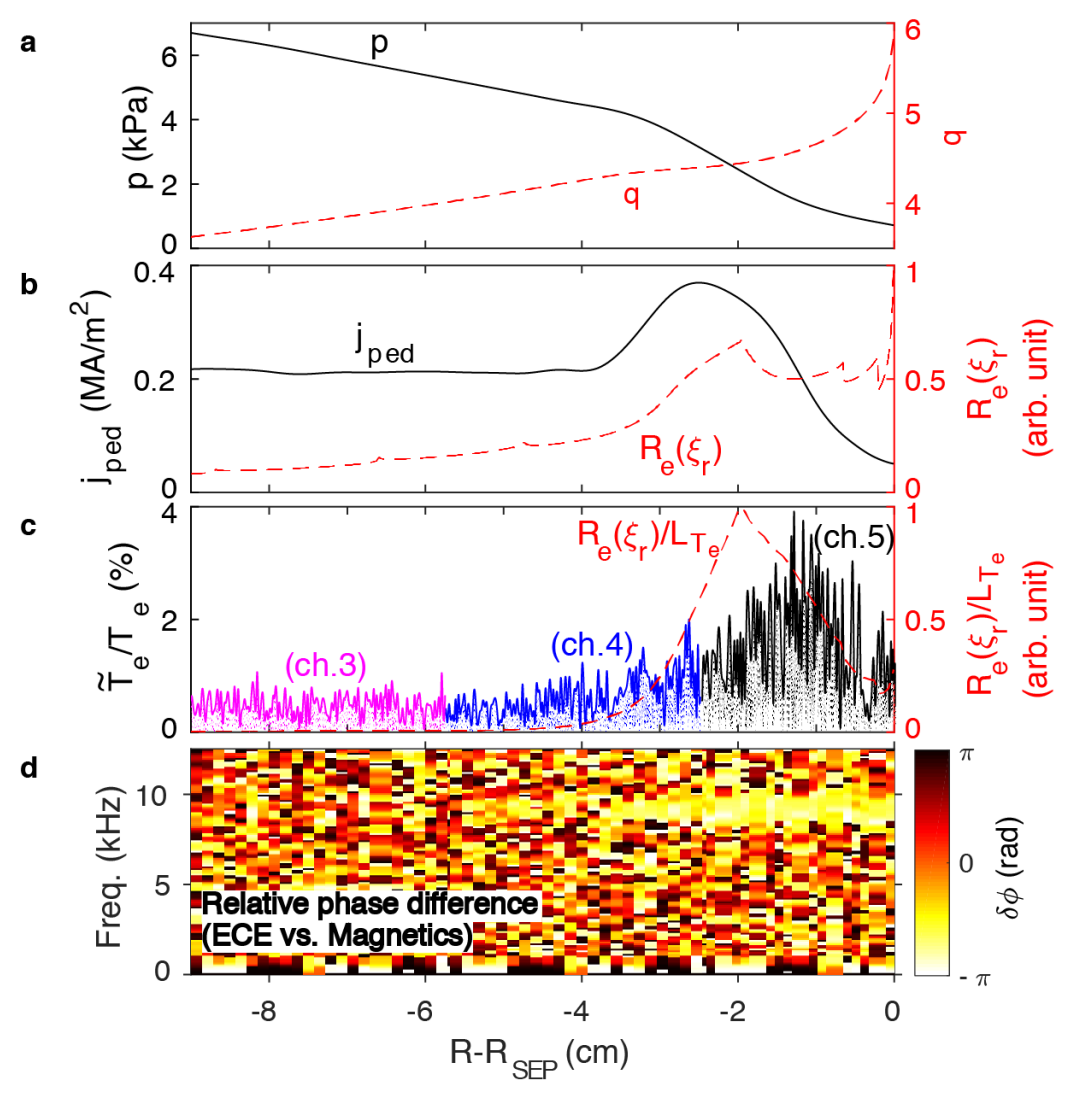

Figure 3 Radial profiles of the QH-mode phase. (a) Pressure and safety factor, (b) pedestal current and linear eigenfunction of the unstable PBM (peeling-ballooning mode) obtained with the MARG2D code, (c) normalized electron-temperature fluctuations measured by the ECE for the fundamental component of the EHOs and the product of the linear eigenfunction times the inverse gradient scale length, and (d) the frequency spectrum of the relative phase difference between the ECE and the magnetic diagnostics (saddle coil). These radial structures were obtained during the GAP scan shown in Fig. 2 for discharge E042868 $(t=6.325-6.565$ s), mapped onto the single-time equilibrium at $\mathrm{t}=6.5 \mathrm{~s}$.

Dynamical steady state of the peeling mode. Examining Fig. 3 (c) in more detail, we note that the reconstructed radial structure of the $1 \mathrm{f}_{\mathrm{EHO}}$ mode is not smooth, unlike a Gaussian shape. Indeed, the envelope of the $1 \mathrm{f}_{\mathrm{EHO}}$ mode seen in the ECE data $\left(\widetilde{T}_{e}^{E n v}\right)$, at which a narrow bandpass filter $\left(\mathrm{f}_{\mathrm{BPF}}=\right.$ 9.53-9.65 kHz) was applied for the raw data, exhibits repeated growth and damping cycles in the span of a few milliseconds (the corresponding frequency is about $\mathrm{f}_{\text {envelope }} \sim 170 \mathrm{~Hz}$ ) at a fixed 
measurement position (e.g., $\mathrm{R}-\mathrm{R}_{\mathrm{SEP}} \sim-1 \mathrm{~cm}$ ), as illustrated in Fig. 4 (a). The oscillation amplitude is the same as that of $\tilde{T}_{e}^{E n v}$. at the LFS, as can be seen in the $1 \mathrm{f}_{\mathrm{EHO}}$ envelope signals from both the FIR-U1 (HFS) and the FIR-U2 (Top/Bottom) data at almost the same frequency $\left(\sim \mathrm{f}_{\text {envelope }}\right)$, as shown in Figs. 4 (b) and (d). The most important point is that the same oscillation at the frequency $\sim \mathrm{f}_{\text {envelope }}$ can be seen in the "mean" temperature gradient, $\nabla \widetilde{T}_{e}^{M e a n} \equiv \Delta \widetilde{T}_{e}^{E C E} / \Delta R^{E C E}$, as shown in Figs. 4 (c) and (d). Here, the values of $\Delta \widetilde{T}_{e}^{E C E}$ are obtained by subtracting the ECE data at $\mathrm{R}-\mathrm{R}_{\mathrm{SEP}} \sim-1 \mathrm{~cm}$ from that at $\mathrm{R}-\mathrm{R}_{\mathrm{SEP}} \sim-4 \mathrm{~cm}\left(\triangle R^{E C E}=3 \mathrm{~cm}\right)$ and applying a bandpass filter over the frequency range $\mathrm{f}_{\mathrm{BPF}}=100-300 \mathrm{~Hz}$.
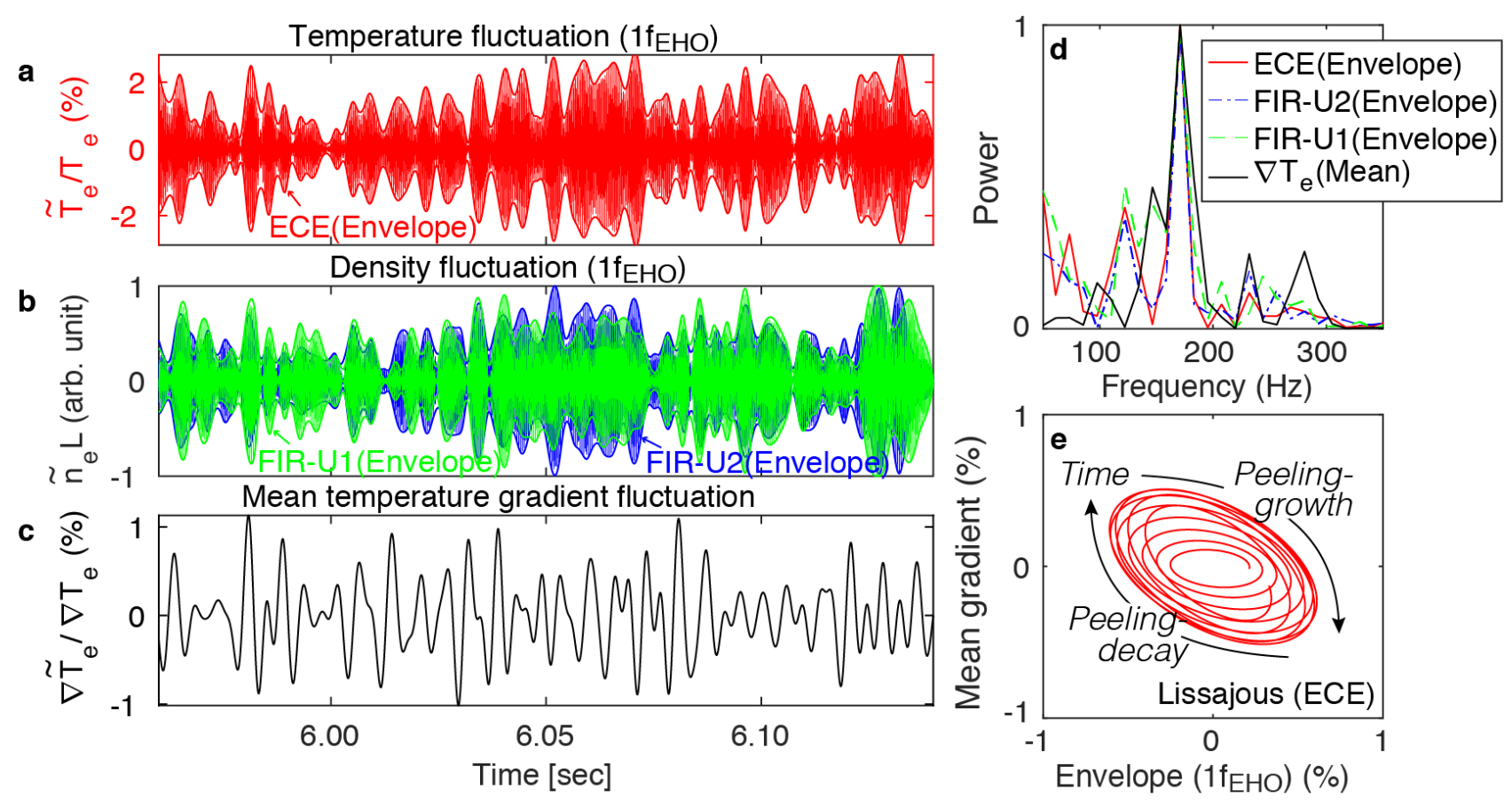

Figure 4 Dynamical steady state of the peeling mode in QH-mode (discharge E042870). Temporal behavior of the fluctuations (a) of the temperature measured by the ECE, (b) of the density measured by FIR-U1 (green) and -U2 (blue), and (c) of the mean temperature gradient (discharge E042870). A bandpass filter ( $f_{B P F}=9.53-9.65 \mathrm{kHz}$ ) around the fundamental component of the EHOs ( $1 \mathrm{f}_{\mathrm{EHO}}$ ) was applied to the raw data in (a) and (b), while a bandpass filter with $\mathrm{f}_{\mathrm{BPF}}=0.1-0.3 \mathrm{kHz}$ was applied in (c). Frequency spectra (d) for the envelope of the data shown in (a)(b), and for the mean temperature gradient data shown in (c). (e) The relationship between fluctuations of the mean temperature gradient (Fig. 4c) and the envelope signal of the $1 \mathrm{f}_{\mathrm{EHO}}$ mode (Fig. 4a) from the ECE data after applying an additional bandpass filter around $\mathrm{f}_{\text {envelope }}=170 \pm 15 \mathrm{~Hz}$ traces a Lissajous figure. The time-window for (d) $-(\mathrm{e})$ is $\mathrm{t}=6.01-6.07 \mathrm{sec}$.

Note that the experimental identification described above was made possible because the EHOs were in a state close to that of a linear mode, with almost no higher harmonics, although the observation of a QH-mode phase that remained stationary longer than its MHD stability growth rate seems strange from a common-sense point of view. Thus, there must be a mechanism to keep the 
peeling mode from growing explosively. Here, this is provided by a "dynamical" stationary phase that has a timescale of variation of a few $\mathrm{ms}\left(\sim 1 / \mathrm{f}_{\text {envelope}}\right)$, which is a much longer span of time than that of the peeling mode: $1 / \mathrm{f}_{\mathrm{EHOs}} \sim \boldsymbol{O}(0.1 \mathrm{~ms})$. We conclude that the peeling mode (which has almost no higher-harmonic components) has been identified as a new "dynamical" steady state of a QHmode phase that does not have any large ELMs.

This dynamical steady state is realized as a limit-cycle oscillation, as shown by the relationship between $\nabla T_{e}^{M e a n}$ (the mean temperature gradient) and $\widetilde{T}_{e}^{E n v}$. (the envelope of the $1 \mathrm{f}_{\mathrm{EHO}}$ mode): as illustrated in Fig. 4 (e), the change in $\nabla T_{e}^{\text {Mean }}$ follows the change in $\tilde{T}_{e}^{E n v}$. Once the peeling mode grows (observed as increments of $\tilde{T}_{e}^{E n v}$.), the mean gradient becomes small. The decrease in the mean gradient suppresses the peeling mode growth (seen in the decrease in $\tilde{T}_{e}^{E n v}$ ), which results in the recovery of the pedestal structure toward the original state. Then, the peeling mode starts to grow again, and the cycle repeats.

\section{Discussion}

This limit-cycle was modeled in Ref. 27 . Noting that the oscillation period [e.g., $1 / \mathrm{f}_{\text {envelope }} \sim \boldsymbol{O}(1$ $\mathrm{ms})$ ] is much shorter than the current-diffusion time [e.g., $\boldsymbol{O}(1 \mathrm{sec})]$, the coupled dynamics between the peeling mode amplitude and the edge pressure gradient was studied by assuming that the magnetic structure (edge current, magnetic-shear parameter, etc.) is constant during this oscillation. We found that a limit-cycle oscillation of the paired mode amplitude and edge gradient can emerge if the stability boundary has a form like that shown in Fig. 2(c); i.e., the peeling mode is unstable in the higher-gradient domains across the stability boundary. Estimating the incremental loss rate of the edge gradient due to the peeling mode as $\tau_{\text {peel }} \sim \mathrm{d}^{2} / \Gamma \xi^{2}$ — where $d$ is the scale length of the gradient of the edge pedestal and $\Gamma$ and $\xi$ are the decorrelation rate and the radial-oscillation length of the peeling mode, respectively - the characteristic timescale of the limit-cycle oscillation is $2 \pi f_{\mathrm{LCO}} \mathrm{Cal}_{\sim}\left(\gamma_{0} / \tau_{\text {peel }}\right)^{1 / 2}$, where $\gamma_{0}$ is the characteristic growth rate of the linear peeling mode. If one chooses $\xi \sim \boldsymbol{O}(1 \mathrm{~mm})$ (which needs further experimental verification), $d \sim 2 \mathrm{~cm}$, and $\Gamma / 2 \pi \sim 0.5 \mathrm{kHz}$ as characteristic estimates from the observations, one obtains $1 / \tau_{\text {peel }} \sim \boldsymbol{O}\left(10 \mathrm{~s}^{-1}\right)$. Using a typical value - say, $\gamma_{0} \sim 10^{5}$ $\mathrm{s}^{-1}$ - one finds $\left(\gamma_{0} / \tau_{\mathrm{peel}}\right)^{1 / 2} \sim \boldsymbol{O}\left(10^{3} \mathrm{~s}^{-1}\right)$ and $f_{\mathrm{LCO}} \mathrm{Cal} \sim \boldsymbol{O}(200 \mathrm{~Hz})$. The observations are quantitatively consistent with this new model of limit-cycle oscillations, especially for the expected order of

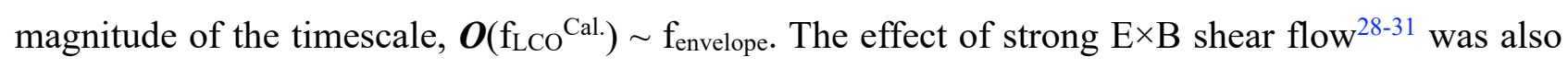
discussed in Ref. 27, and the result is qualitatively unchanged.

In conclusion, we have presented the first evidence of the peeling mode for EHOs seen in the peripheral region of the plasma for the QH-mode in JT-60U, and it meets the five criteria described above. New information about the saturation mechanism that enables the EHOs to peel off the pedestal (without the effect growing explosively) is also shown. In particular, the dominant 
fundamental mode of the EHOs is observed to repeat cycles of growth and damping, in association with changes in the mean temperature gradient, on the order of a few $100 \mathrm{~Hz}$. This observation is quantitatively consistent with the new dynamical quasi-linear steady state model of limit-cycle oscillations whether or not a strong $\mathrm{E} \times \mathrm{B}$ shear flow exists. This sheds further light on the "peeling nature" of both laboratory plasmas and astronomical plasmas (such as ELM events in magneticconfinement-fusion plasmas and solar/stellar flares).

\section{Methods}

\section{QH-mode operation on JT-60U.}

The JT-60U tokamak is a single null divertor tokamak device having the plasma major radius $\mathrm{R}_{\mathrm{P}}=$ $3-3.5 \mathrm{~m}$, the plasma minor radius $\mathrm{a}_{\mathrm{P}}=0.6-1.1 \mathrm{~m}$ and the maximum toroidal magnetic field $\mathrm{B}_{\mathrm{T}} \leq 4$ $\mathrm{T}$ at major radius $\mathrm{R}=3.32 \mathrm{~m}$. The $\mathrm{QH}-$ mode plasma on JT-60U can be reproduced at a lower electron density regime (e.g., Greenwald density fraction of up to $\sim 0.4$ ) under a specific cross-sectional shape of the plasma having elongation $\kappa=1.47$ and triangularity $\delta=0.27$ (the total plasma volume of 60 $\mathrm{m}^{3}$ ) with plasma current $\mathrm{I}_{\mathrm{p}}=1.0 \mathrm{MA}, \mathrm{B}_{\mathrm{T}}=2.7 \mathrm{~T}$, and safety factor at the $95 \%$ flux surface $\mathrm{q}_{95} \sim 4$.

\section{Fluctuation measurements for edge-harmonic oscillations (EHOs).}

The temporal evolution of the multi-mode structure FFT spectra during a stationary, long-sustained QH-mode is obtained from the electron-cyclotron-emission radiometer (ECE) used for local electrontemperature measurements at the LFS and from the FIR laser interferometers used to obtain lineintegrated electron density measurements along two vertical chords, which pass through the peripheral region of the HFS plasma (FIR-U1) and from top to bottom of the plasma center (FIR-U2), respectively.

\section{Radial profile measurements for toroidal plasma flow and radial electric field.}

In the study of the JT-60U tokamak for the fiscal year FY2003 experimental campaign, the radial profiles for the density, temperature, and poloidal/toroidal plasma flows of fully stripped carbon impurity ions are measured by means of the Charge eXchange Recombination Spectroscopy (CXRS) diagnostic method with time resolution up to $60 \mathrm{~Hz}$ at 59 spatial points (23 toroidal and 36 poloidal viewing chords). With regard for determining the $\mathrm{E}_{\mathrm{r}}$ structure at the pedestal region, we measured the pressure gradient, and plasma velocity perpendicular to the magnetic field, and the $\mathrm{E}_{\mathrm{r}}$ was evaluated by the radial force balance equation.

\section{References}

1. Connor, J. W. Edge-localized modes - physics and theory. Plasma Phys. Controlled Fusion 40, 
531 (1998).

2. Evans, T. et al. Edge stability and transport control with resonant magnetic perturbations in collisionless tokamak plasmas. Nature Phys 2, 419-423 (2006).

3. Kirk, A. et al. Evolution of Filament Structures during Edge-Localized Modes in the MAST Tokamak. Phys. Rev. Lett. 96, 185001 (2006).

4. Fundamenski, W. et al. On the relationship between ELM filaments and solar flares. Plasma Phys. Control. Fusion 49 R43 (2007).

5. Shibata, K. \& Magara, T. Solar Flares: Magnetohydrodynamic Processes. Living Rev. Sol. Phys. 8, 6 (2011).

6. Shimada, M. et al. Chapter 1: Overview and summary. Nucl. Fusion 47, S1-S17 (2007).

7. Doyle, E.J. et al. Chapter 2: Plasma confinement and transport. Nucl. Fusion 47, S18-S127 (2007).

8. Federici, G. et al. Overview of EU DEMO design and R\&D activities. Fusion Engineering and Design 89, 882-889 (2014).

9. Tobita, K. et al. Japan's Efforts to Develop the Concept of JA DEMO During the Past Decade, Fusion Science and Technology, 75, 372-383 (2019).

10. Snyder, P. B. et al. Edge localized modes and the pedestal: A model based on coupled peelingballooning modes. Physics of Plasmas 9, 2037 (2002).

11. Kamiya, K. et al. Edge localized modes: recent experimental findings and related issues. Plasma Phys. Control. Fusion 49 S43-S62 (2007).

12. Loarte, A. et al. Progress on the application of ELM control schemes to ITER scenarios from the non-active phase to DT operation. Nucl. Fusion 54, 033007 (2014).

13. Leonard, A. W. Edge-localized-modes in tokamaks. Phys. Plasmas 21, 090501 (2014).

14. Burrell, K. H. et al. Quiescent H-mode plasmas in the DIII-D tokamak. Plasma Phys. Control. Fusion 44 A253-A263 (2002).

15. Oyama, N. et al. Energy loss for grassy ELMs and effects of plasma rotation on the ELM characteristics in JT-60U. Nucl. Fusion 45 871-881 (2005).

16. Suttrop, W. et al. Studies of the 'Quiescent H-mode' regime in ASDEX Upgrade and JET. Nucl. Fusion 45 721-730 (2005).

17. Osborne, T. H. et al. Edge stability of stationary ELM-suppressed regimes on DIII-D. Journal of Physics: Conference Series 123012014 (2008).

18. Burrell, K. H. et al. Quiescent H-Mode Plasmas with Strong Edge Rotation in the Cocurrent Direction. Phys. Rev. Lett. 102, 155003 (2009).

19. Chen, Xi. et al. Rotational shear effects on edge harmonic oscillations in DIII-D quiescent H- 
mode discharges. Nucl. Fusion 56, 076011 (2016).

20. Liu, F. et al. Nonlinear MHD simulations of QH-mode DIII-D plasmas and implications for ITER high Q scenarios. Plasma Phys. Control. Fusion 60, 014039 (2018).

21. Snyder, P. B. et al. Stability and dynamics of the edge pedestal in the low collisionality regime: physics mechanisms for steady-state ELM-free operation. Nucl. Fusion 47, 961-968 (2007).

22. Aiba, N. et al. Impact of rotation and ion diamagnetic drift on MHD stability at edge pedestal in quiescent H-mode plasmas. Nucl. Fusion 60, 092005 (2020).

23. Aiba, N. et al. Stabilization of kink/peeling modes by coupled rotation and ion diamagnetic drift effects in QH-mode plasmas in DIII-D and JT-60U. FEC2020 synopsis.

24. Aiba, N. et al. Analysis of ELM stability with extended MHD models in JET, JT-60U and future JT-60SA tokamak plasmas. Plasma Phys. Control. Fusion 60, 014032 (2018).

25. Wesson, J.A. Hydromagnetic stability of tokamaks. Nucl. Fusion 18, 87 (1978).

26. Manheimer, W. M. \& Lashmore-Davis, C. N. MHD and Microinstabilites in Confined Plasma (Adam Hilger, 1989).

27. Itoh, K. et al. On the possibility of limit-cycle-state of peeling mode near stability boundary in the quiescent H-mode. Plasma Phys. Control. Fusion 63, 025002 (2021).

28. Guo, Z. B. \& Diamond, P. H. From Phase Locking to Phase Slips: A Mechanism for a Quiescent H mode. Phys. Rev. Lett. 114, 145002 (2015).

29. Chen, Xi. et al. Bifurcation of quiescent H-mode to a wide pedestal regime in DIII-D and advances in the understanding of edge harmonic oscillations. Nucl. Fusion 57, 086008 (2017).

30. Wilks, T.M. et al. Scaling trends of the critical $\mathrm{E} \times \mathrm{B}$ shear for edge harmonic oscillation onset in DIII-D quiescent H-mode plasmas. Nucl. Fusion 58, 112002 (2018).

31. Brunetti, D. et al. Excitation Mechanism of Low-n Edge Harmonic Oscillations in Edge Localized Mode-Free, High Performance, Tokamak Plasmas. Phys. Rev. Lett. 122, 155003 (2019).

\section{Acknowledgements}

The authors deeply appreciate the continued research and operational efforts of the entire JT-60 team. Authors acknowledge the partial support by Grant-in-Aid for Scientific Research (JP 15K06657, JP 15H02155, JP 16H02442) and collaboration programs between QST and universities and of the RIAM of Kyushu Univ., and by Asada Science Foundation. Authors wish to dedicate this article to the memory of late Prof. Sanae-I. Itoh.

\section{Author contributions}

K.K analyzed the data. K.I provided the theoretical models. N.A and M.H analyzed the MHD stability. 
K.K, K.I, and N.A discussed the model validation. K.K, N.O, and A.I discussed the diagnostics accuracy for the fluctuation measurements. K.K and K.I wrote the main manuscript text and all authors reviewed the manuscript.

\section{Additional information}

Competing financial interests: The authors declare no competing financial interests. 

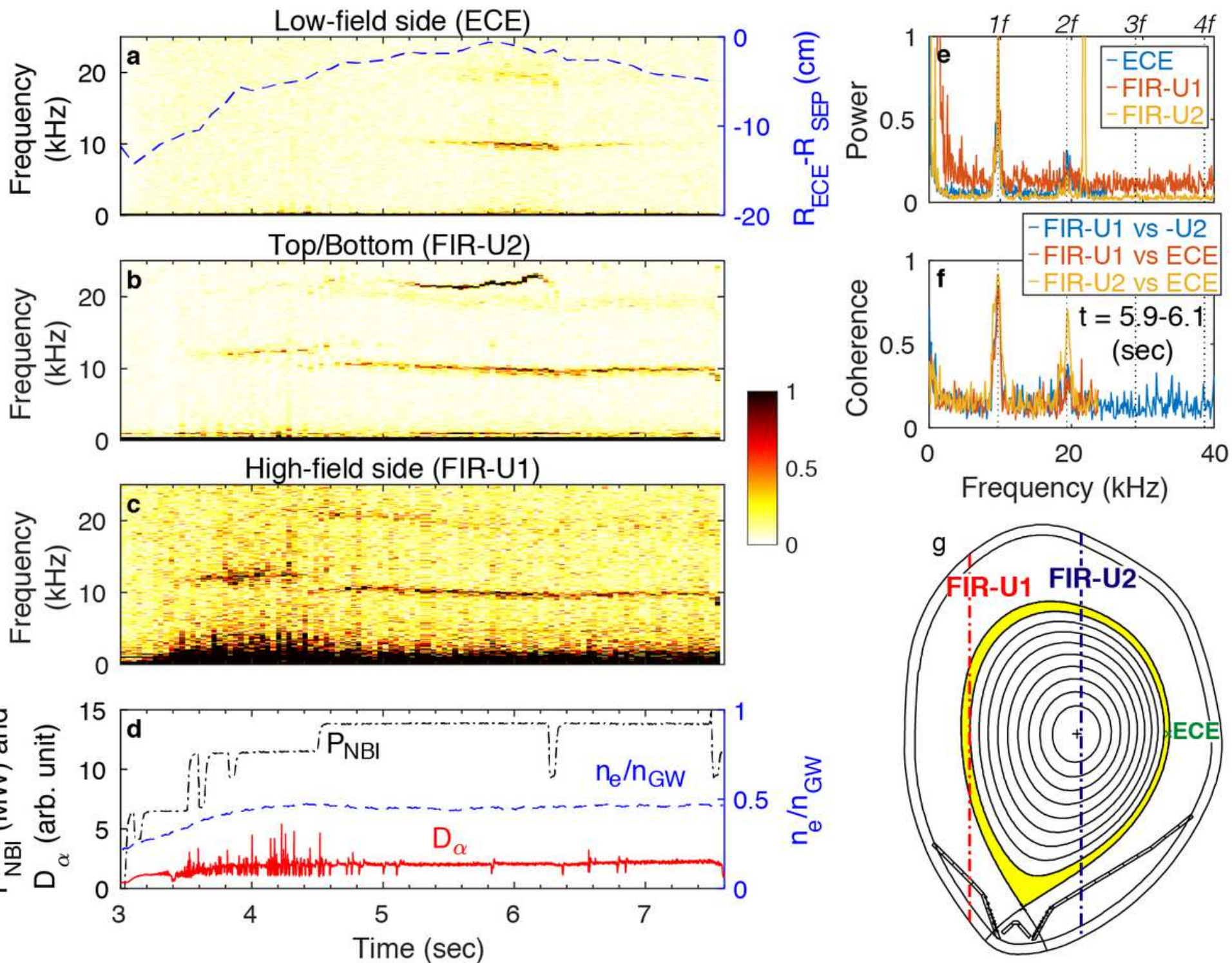

Figure 1

Quiescent H-mode shot (discharge E042870). Fourier analysis of signals (normalized at $\mathrm{t}=6.0 \mathrm{~s})(\mathrm{a})$ from the ECE at the LFS, together with its measurement position relative to the separatrix, and from the FIR laser interferometers for (b) the Top/Bottom (FIR-U2) and (c) the HFS (FIR-U1) chords. (d) The total NBI power, Da emission from the divertor, and line-averaged electron density normalized by the Greenwald density. The frequency dependences of (e) the normalized power spectra for the ECE, FIR-U1, and -U2 and (f) their respective coherences evaluated at $t=5.9-6.1 \mathrm{~s}$. $(\mathrm{g})$ Magneto-hydrodynamic equilibrium (from the FBEQU-code) and the diagnostic locations at $t=6.0 \mathrm{~s}$. 

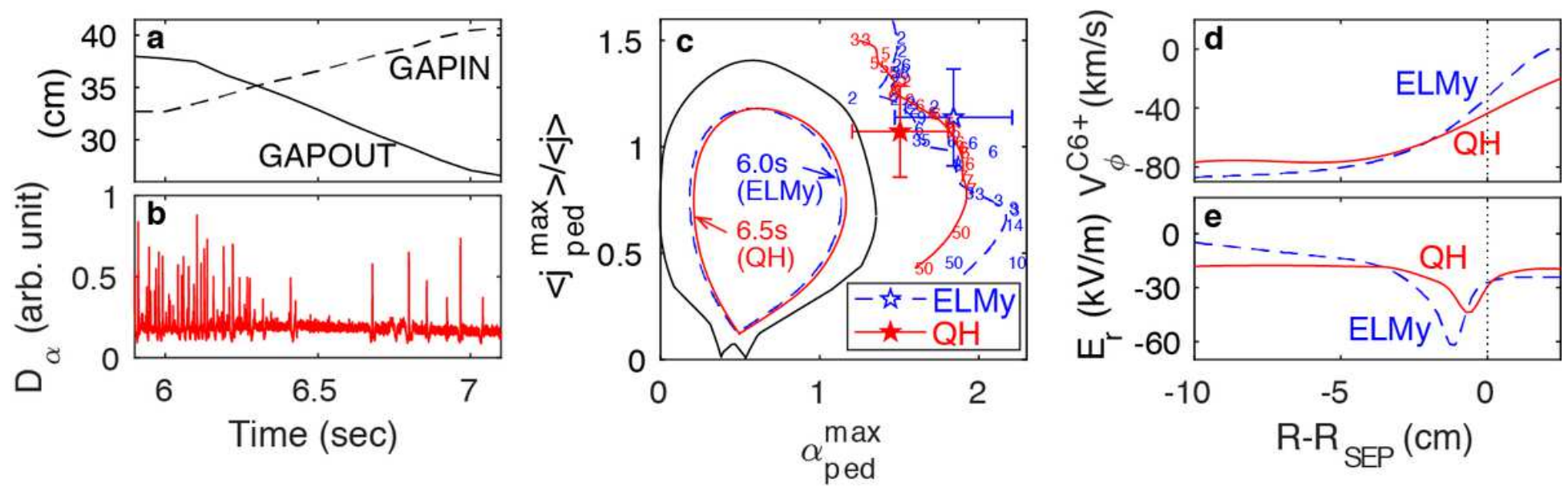

Figure 2

Transition from ELMy to the QH-mode in a gap scan during discharge E042868. (a) GAPOUT and GAPIN, and (b) Da emission from the divertor. Comparisons between $t=6.0 \mathrm{~s}$ (ELMy: blue dashed line) and $6.5 \mathrm{~s}$ (QH: red solid line) for (c) the MHD stability diagram in the normalized pedestal current ( $\nabla$ ped.max $/<j>$ ) and pressure (『ped.max) plane, showing the MHD equilibria, and the radial profiles of (d) the toroidal flow and (e) the radial electric field. 
a

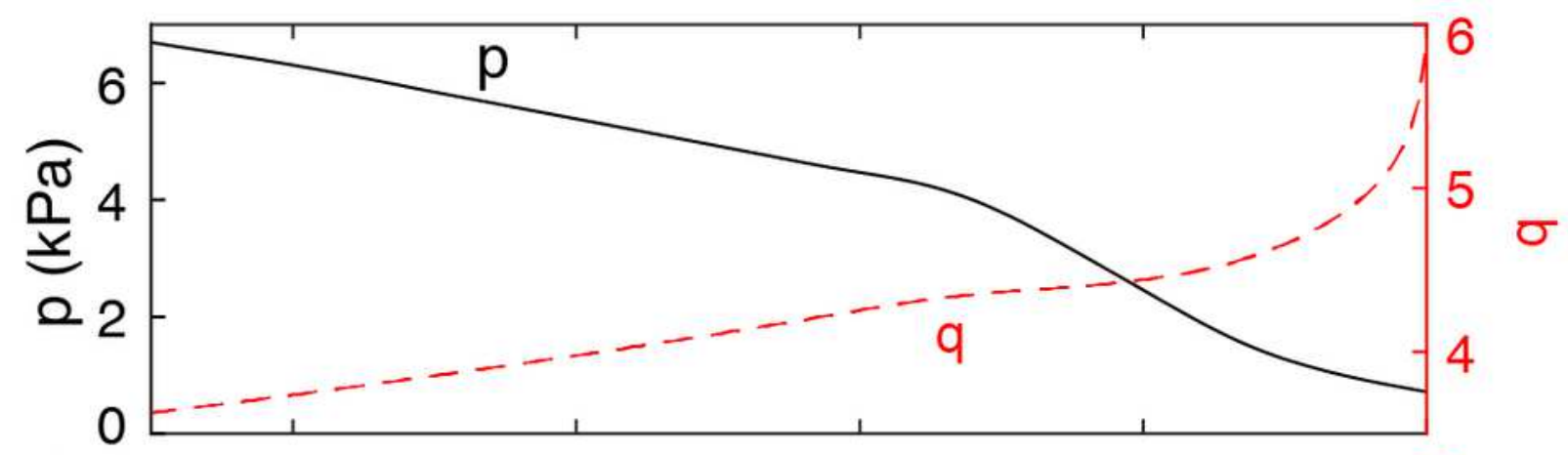

b

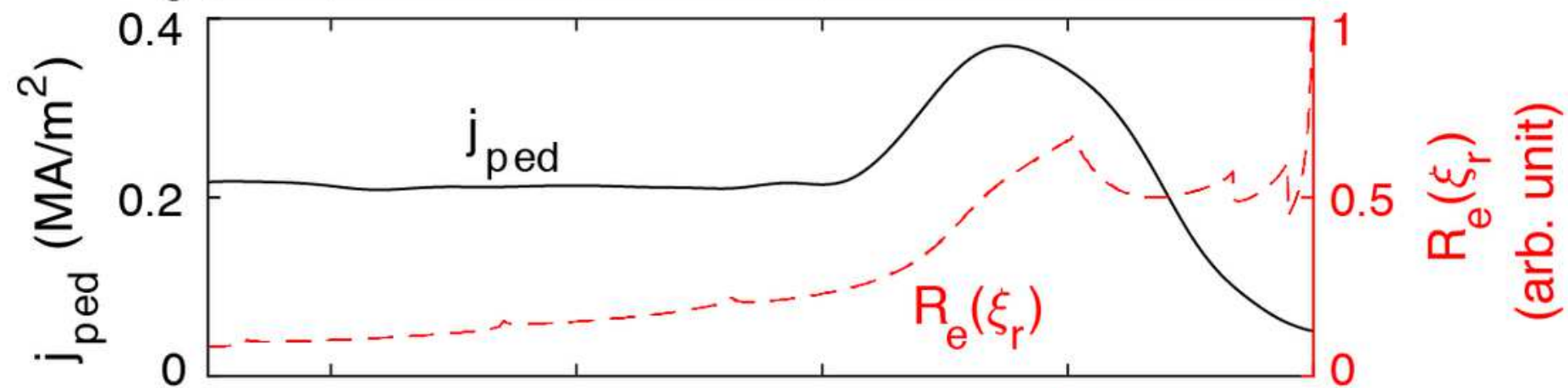

C
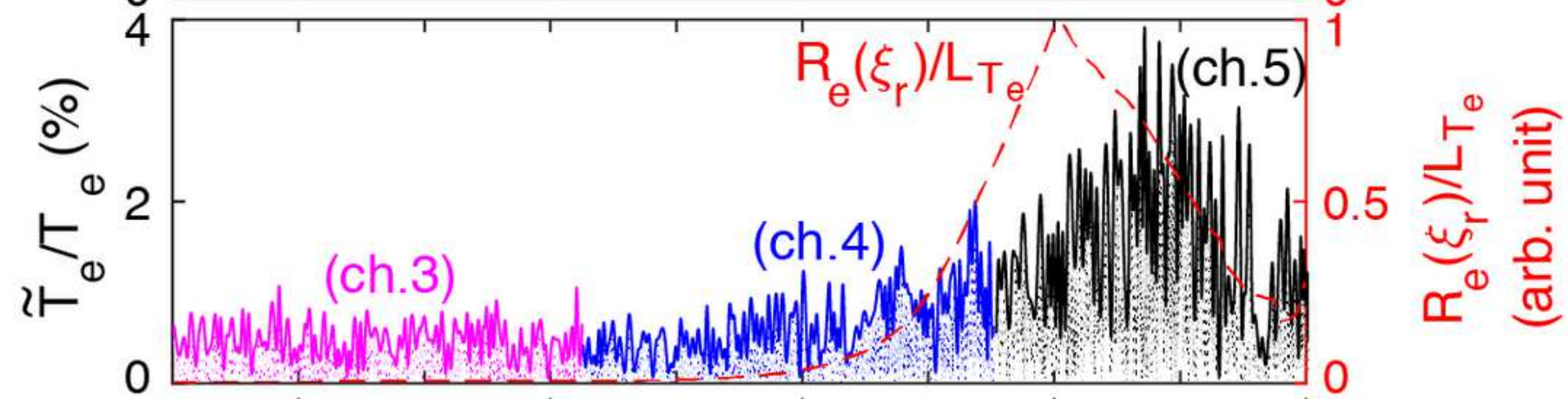

d

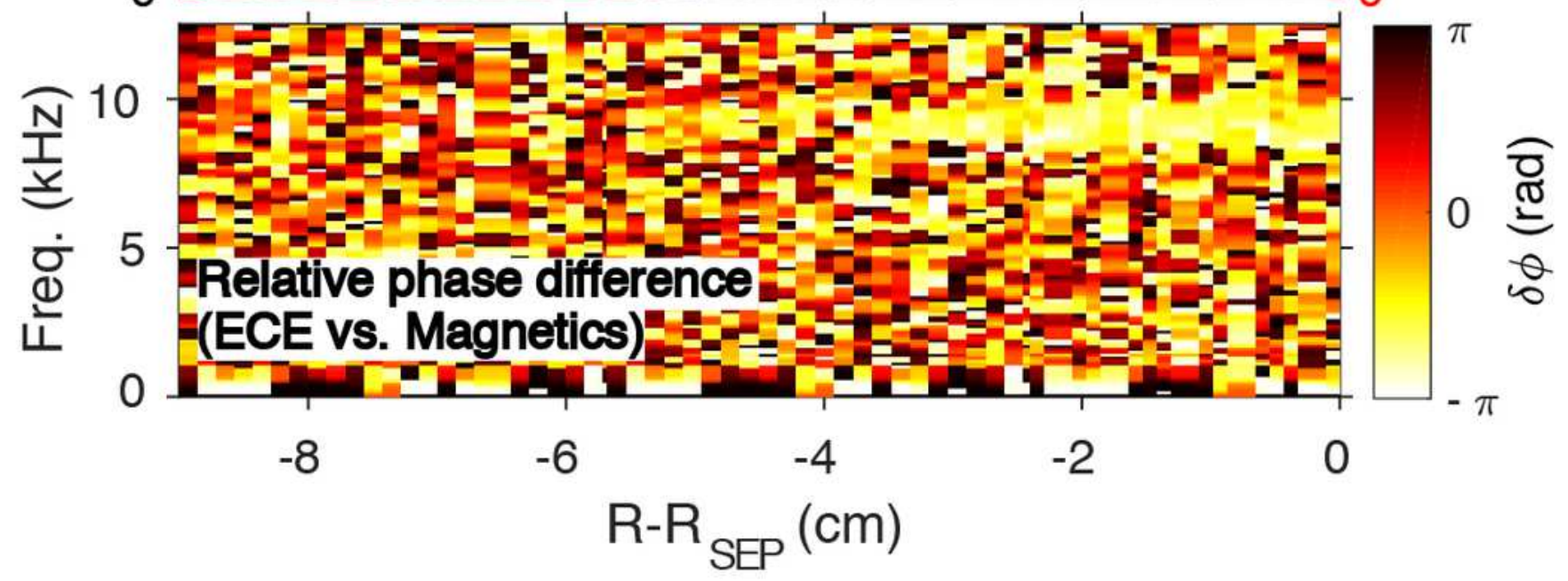

Figure 3

Radial profiles of the QH-mode phase. (a) Pressure and safety factor, (b) pedestal current and linear eigenfunction of the unstable PBM (peeling-ballooning mode) obtained with the MARG2D code, (c) normalized electron-temperature fluctuations measured by the ECE for the fundamental component of the EHOs and the product of the linear eigenfunction times the inverse gradient scale length, and (d) the frequency spectrum of the relative phase difference between the ECE and the magnetic diagnostics 
(saddle coil). These radial structures were obtained during the GAP scan shown in Fig. 2 for discharge E042868 $(t=6.325-6.565 \mathrm{~s})$, mapped onto the single-time equilibrium at $\mathrm{t}=6.5 \mathrm{~s}$.
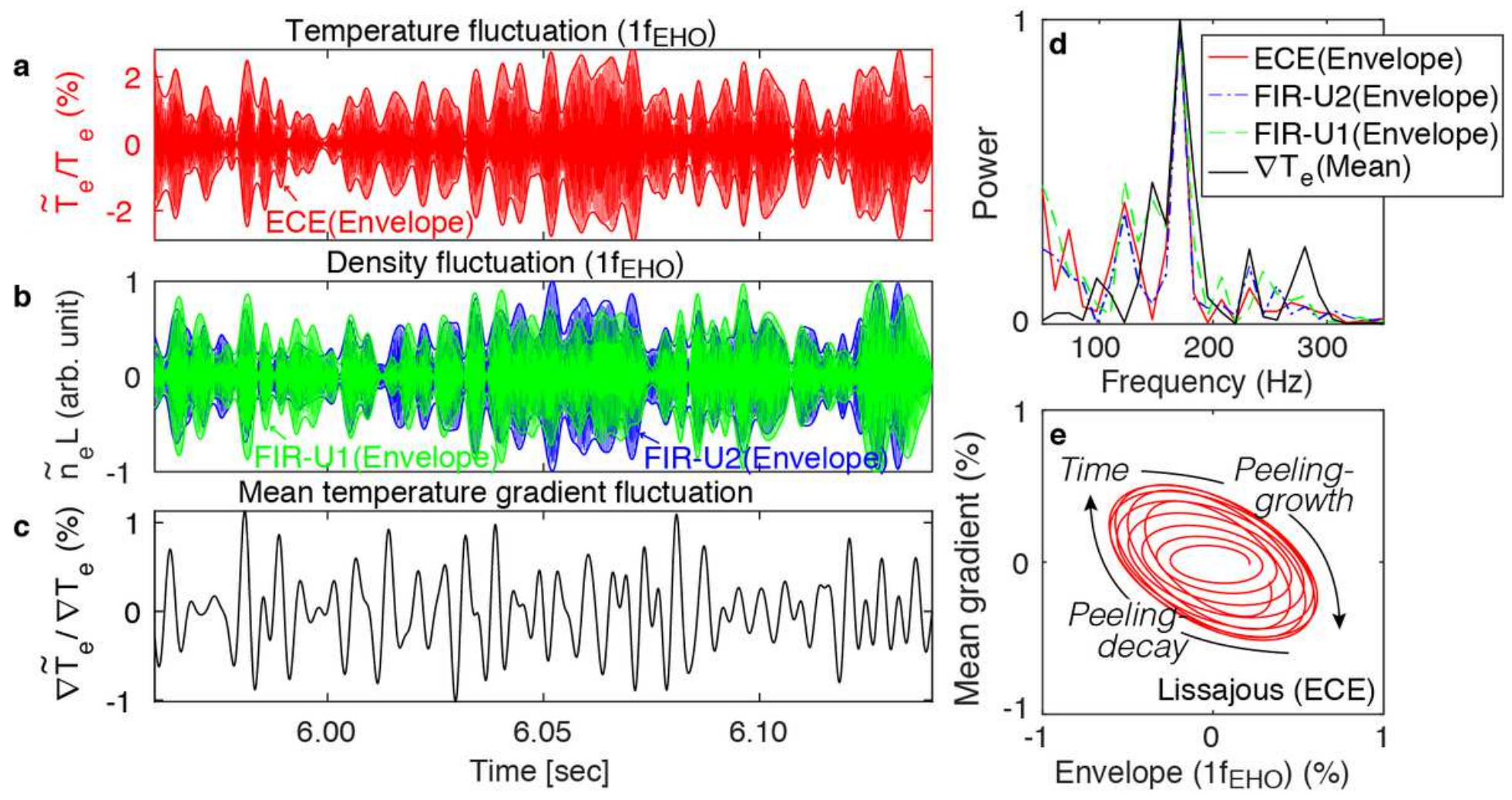

Figure 4

Dynamical steady state of the peeling mode in QH-mode (discharge E042870). Temporal behavior of the fluctuations (a) of the temperature measured by the ECE, (b) of the density measured by FIR-U1 (green) and $-\mathrm{U} 2$ (blue), and (c) of the mean temperature gradient (discharge E042870). A bandpass filter (fBPF = 9.53-9.65 kHz) around the fundamental component of the EHOs (1 fEHO) was applied to the raw data in (a) and (b), while a bandpass filter with fBPF $=0.1-0.3 \mathrm{kHz}$ was applied in (c). Frequency spectra (d) for the envelope of the data shown in (a)-(b), and for the mean temperature gradient data shown in (c). (e) The relationship between fluctuations of the mean temperature gradient (Fig. 4c) and the envelope signal of the $1 \mathrm{fEHO}$ mode (Fig. 4a) from the ECE data after applying an additional bandpass filter around fenvelope $=170 \pm 15 \mathrm{~Hz}$ traces a Lissajous figure. The time-window for $(d)-(e)$ is $t=6.01-6.07 \mathrm{sec}$. 